\title{
ANALYSIS OF DIFFERENT DYNAMIC MODELING TECHNIQUES FOR USE IN SIMULATION ENVIRONMENT (HARDWARE IN THE LOOP-HIL)
}

\author{
Eduardo Torrigo ${ }^{1}$, Alexandre Kawamoto ${ }^{2}$, Henrique Manguino $^{2}$ e Pedro Rossetti ${ }^{2}$ \\ ${ }^{1}$ MWM Motores Diesel \\ ${ }^{2}$ ETAS \\ E-mails: eduardo.torrigo@navistar.com.br, alexandre.kawamoto@etas.com, \\ henrique.meira@etas.com, pedro.rossetti@etas.com
}

\begin{abstract}
Many times the most effective way to develop and test a new engine application is to install the engine into the real vehicle or machine, however depending on the complexity and number of systems to be tested then the required effort and investment can be very high that in some cases such approach could be considered economically unfeasible specially if applied for a small production volume (units/year).

Hardware in the Loop (HIL) equipments have the ability to emulate the engine and vehicle behavior (static and dynamic) by sensing the engine actuators connected to HIL and stimulating the engine and vehicle sensors according to signals calculated by representative engine/vehicle models that can provide a realistic well controlled virtual environment for vehicle systems test. In some cases it can be more efficient than the real vehicle test environment because of elimination of random test noise sources.

To support engineers to build engine models for HIL simulation this work shows details of a complex airpath modeling of a modern Euro V EGR/VGT engine using three different modeling techniques: 1) Frequency response; 2) Autoregressive exogenous (ARX); 3) Statistical Learning with Nonlinear Autoregressive Exogenous approach (NARX).

Detailed methodology procedures are presented describing since the data collection tests, data analysis, model determination and simulation. At the end three methodologies are compared with regards to model accuracy and complexity of use.
\end{abstract}

\section{INTRODUCTION}

One of the most complex systems to model is the engine airpath (intake manifold pressure and amount of fresh air available). Emission control and vehicle drivability in a diesel engine relies considerable on the airpath behavior therefore accurate models of such system are an important part of the engine simulation in HIL. Engine airpath model must consider simultaneously both variables (pressure and fresh air mass flow) and at least its direct control actuators (VGT turbo control and EGR valve opening) as seen on Figure 1. 


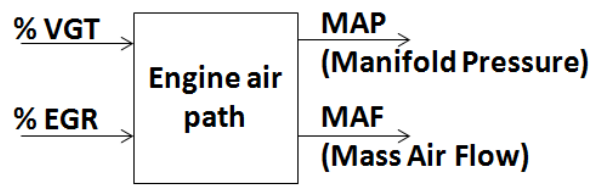

Figure 1: Input / output representation

As both actuators (EGR and Turbo) share the same energy from the exhaust gases a model that considers such coupling effect can better describe the engine airpath behavior in simulation environment like proposed by Yue-Yun [1]. The coupling effect can be modeled using the diagram seen in Figure 2.

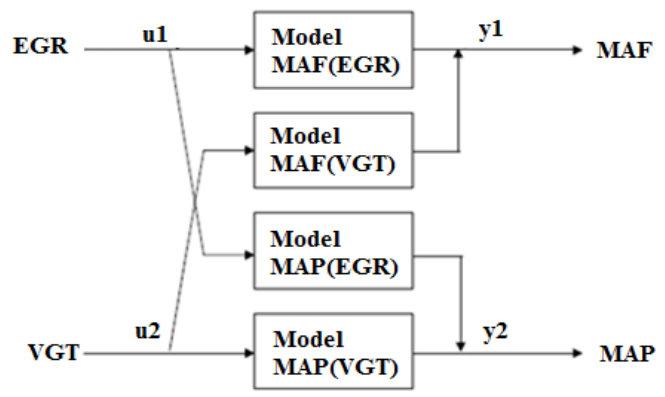

Figure 2: Process model with couplings ( 2 direct and 2 indirect couplings)

Engine airpath variables have highly non-linear behavior [2] however they can be approximately by a linear functions around a specific operation point and such approach can be valid in some cases for limited amplitude actuator variation (EGR or VGT) as described by Yue-Yun [1]. Each small block seen above will contain a dynamic model that according to Alfieri [3] can be written in terms of a transport delay associated with a linear transfer function as seen on Figure 3.

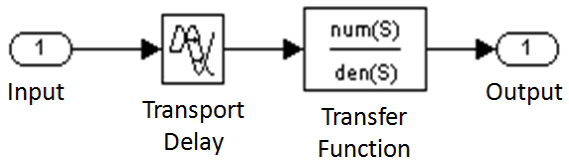

Figure 3: Input-Output model

Transport delay can be determined by step stimulus test at the actuators position control. Linear behavior can be tested and confirmed by applying a position sweep (e.g. +/- 10\%) in each actuator (EGR and VGT) around the desired engine operation point (load and speed) while measuring the controlled variables (Intake manifold pressure and fresh air mass flow) similarly as proposed by Yue-Yun [1].

This work will build input-output models by three modeling approaches: Frequency response (FR); Autoregressive exogenous (ARX) and Statistical Learning with Nonlinear Autoregressive Exogenous approach (NARX). First two methodologies will use MATLAB tool while the third methodology will be handled by ASCMO dynamic modeling tool (ETAS). At the end three models will be implemented into SIMULINK environment to compare the results with real measured data from engine. Data plots and numerical model performance index will be calculated for model performance comparison. 


\section{MODELING METHODOLOGIES}

\subsection{Frequency Response Analysis Methodology}

Frequency Response model determination methodology consists of Bode diagram analysis (amplitude plot). Bode diagrams should be built from the plant response when stimulated by persistent sinusoidal signals. Such approach uses graphical analysis whereas the plant measured gain (output / input) on each frequency can be used to build time invariant linear transfer functions [4]. In order to make analysis easier then asymptotes can be added to the graph to determine the main breaking points like low frequency gain, cut frequency and rate of decay (dB/decade).

\subsection{ARX Analysis Methodology}

It can be used autoregressive exogenous (ARX) models to represent engine airpath like suggested by Ortner [6] or similarly as done by Ueno [5]. An example of a second order time discrete ARX model is given in (1).

$$
\begin{array}{cl}
y(k+1)+A_{1} \cdot y(k)+ & A_{2} \cdot y(k-1)=B_{1} \cdot u(k-T)+B_{2} \cdot u(k-T-1) \\
\text { where: } & -y \text { is the output } \\
& -u \text { is the input } \\
& -T \text { is the transport delay } \\
& -A_{n} \text { and } B_{n} \text { are multiplicative coefficients. }
\end{array}
$$

The ARX equation can be adjusted for the engine airpath response by computer programs (e.g. MATLAB). It requires the input and output data recorded in a constant sampling rate and also the transport delay $(\mathrm{T})$ measured by step response test. Input stimulus will be pseudorandom binary signal (it can be generated by MATLAB command "IDINPUT"). The resulting ARX discrete equation (z-domain) can be converted into continuous (s-domain) by means of the MATLAB command "D2C".

\subsection{Statistical Learning and Gaussian modeling}

The Statistical learning which is one of the ASCMO algorithms uses the results of a parameterized radial basis function network as an Input of the Gaussian process (GP) [9]. This methodology trains the model focused on minimizing the total squared error of a weighted approximating function $\mathrm{f}(\mathrm{x})$. The radial basis function method considers that each point could be approximated to a function $\mathrm{g}$ that satisfies (2):

$$
g(x)=\varphi\|x-c\|
$$

$$
\begin{array}{ll}
\text { where: } & -g \text { is the Generic Radial Basis Kernel } \\
& -c \text { is the Center } \\
& -x \text { is the input }
\end{array}
$$

Any function that follows the $\mathrm{g}(\mathrm{x})$ form can be considered a radial basis function. Weighted radial basis functions network can be created using (3):

$$
f(x)=\sum_{i=1}^{n} w_{i} g_{i}(x)
$$


where: $\quad-f(x)$ is the sum of weighted generic $\mathrm{g}_{\mathrm{i}}(\mathrm{x})$

$-w_{i}$ is the Weight

- $n$ is the number of radial basis function

The results of the radial basis function kernels often have a Gaussian distribution, therefore this generic equation $\mathrm{g}(\mathrm{x})$ can be parameterized and replaced as shown below- The final function is represented by:

$$
f(x)=\sum_{i=1}^{n} w_{i} e^{-\frac{1}{2}\left(\frac{|| x-c_{i, 1}||^{2}}{\sigma_{i, 1}^{2}}\right)}
$$

where: $\quad-f(x)$ is the sum of weighted specific $\mathrm{g}_{\mathrm{i}}(\mathrm{x})$ (when it is a Gaussian distribution)

$-\sigma$ is the Standard deviation

\subsection{Nonlinear Auto regression with Exogenous Inputs}

The dynamic modeling tool uses a Nonlinear Auto regression with Exogenous Inputs (NARX). To do it, the core of the modeling tool feedbacks to be able to consider past inputs and outputs. Then the dynamic problem can be transformed in a quasi-stationary relationship using equation (5):

$$
y(k)=f(x 1(k), x 1(k-1), \ldots, x 2(k), x 2(k-1), \ldots, y(k-1), \ldots)
$$

Where " $k$ " indicates the discreet time-step.

These three different methodologies described above (RF, ARX and NARX with GP) will be used to determine models for the same engine airpath, using same available measured data and the quality of each model will be evaluated using Mean Squared Error (MSE) determined as follow.

1.5 Model Adherence Numerical Determination Methodology.

Each transfer function will be evaluated by means of visual inspection in graphs and also by the index MSE shown in the equation (6) [8].

$$
M S E=\frac{\sum_{i=1}^{n}(y i-\hat{y} i)^{2}}{n}
$$

where: $\quad-n$ is the number of samples in the test

- $y i$ is the $i$-th measured output signal

- $\hat{y} i$ is the $i$-th model estimated output signal

MSE is null when the model has perfect matching to measured data.

\section{ENGINE MEASUREMENTS FOR MODELING}

To cover the three modeling methodologies the engine data acquisition experiment should consider the measurement steps below:

- Engine stabilization in the desired operating point for modeling 
- Linearity check (actuator sweep)

- Step stimulus application

- Sinusoidal stimulus application in several frequencies

- Pseudo random binary stimulus (PRBS) application.

\subsection{Engine stabilization}

For airpath modeling the engine can be installed in a steady state dynamometer where the engine speed and load can be stabilized around the desired operation point.

\subsection{Linearity check}

Since the modeling approach here adopted considers that each part of the airpath can be represented by a linear input-output model therefore the linearity check must be done to confirm that this approach is valid. The linearity check consists of applying a wide position sweep at the EGR and VGT actuators to determine if the output variables to be modeled (MAF and MAF) can be approximated by linear models.

\subsection{Stimulus application}

To obtain correct data for modeling then each stimulus (Step, sinusoidal or PRBS) should be applied in one of the actuators at time while the remaining engine control parameters should be kept unchanged in their nominal conditions inherent the chosen engine operation point. During the stimulation the MAF and MAP closed loop control (PI control) must be disabled to keep the actuators fixed in the nominal condition. An example of sinusoidal stimulus application for the VGT modeling can be seen on Figure 4. This stimulation would provide data for MAF and MAP variables modeling as function of VGT position.

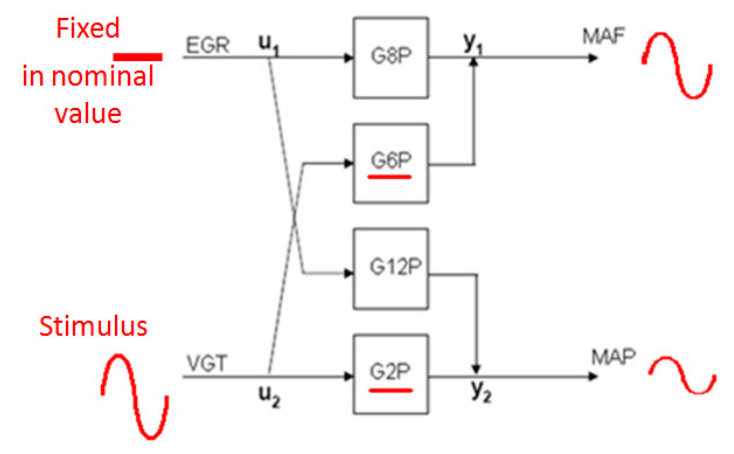

Figure 4: Example of sinusoidal stimulus application into VGT control setting for MAF and MAP modeling as function of turbo actuator.

Same operation should be repeated on the EGR valve while the VGT remains stable in the nominal operation point. Preliminary investigations in the engine at the chosen operation point indicates that manifold pressure (MAP) reacts slowly upon VGT position changes resulting in settling time around 8 seconds while the mass air flow measured (MAF) can return into stabilization from 1 to 2 seconds after EGR valve position changes. These results indicate that the manifold pressure (MAP) modeling using sinusoidal signals should have frequency lower than $15 \mathrm{rad} / \mathrm{sec}$ while the mass air flow (MAF) could reach up to $40 \mathrm{rad} / \mathrm{sec}$. This work considers that the actuators dynamic behavior is included in the global plant behavior and separate models for the actuators characteristics will not be done similarly as 
[6]. Measured data should be stored in time-synchronous computer files for later digital computer processing.

\subsection{Step Stimulus}

Positive step stimulus applied on the engine actuators for dynamic model determination allows observing some important aspects of the plant like overshoot, transport delay and direction of reaction (positive or negative response). Such aspects can be seen on Figure 5 where it was applied a step response at time $=0$ second, for example.

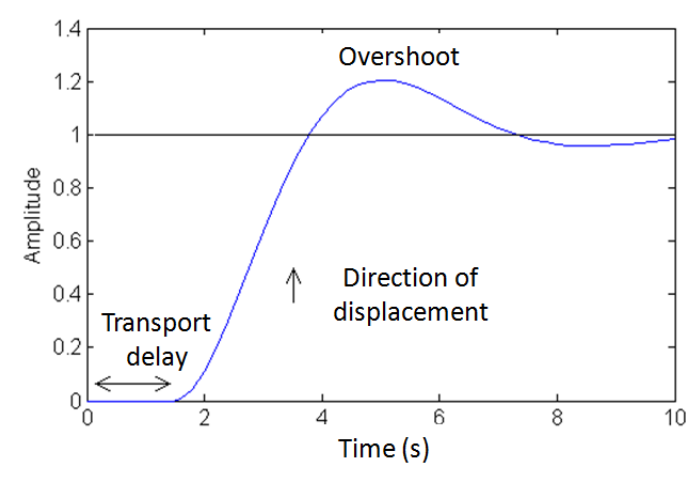

Figure 5: Step response aspects under evaluation

In order to determine the plant behavior then it must be applied individual positive step stimulus on the opening of exhaust gas recirculation valve (EGR) and variable geometry turbine (VGT).

\subsection{Test Management System for stimulation and data acquisition}

It was developed a dedicated application strategy inside ASCET (ETAS tool) software to take over the position control of both actuators (VGT and EGR) from the ECU in order to apply the stimulus as needed for the test while the engine is running.

Engine control unit (ECU) is micro processed equipment dedicated for engine operation which is connected to several sensors (temperature, pressure, speed, flow) and actuators (injectors, turbo, recirculation valve, etc...). For enhanced test capacity it was used a special type of ECU which features an additional communication and control port that is connected to a PC based system for test management and stimulus control. Figure 6 shows the interconnection architecture of the main components for the airpath modeling. Pressure and mass air flow sensors are connected to the ECU while the VGT and EGR actuators are controlled by ECU via CAN messages. 


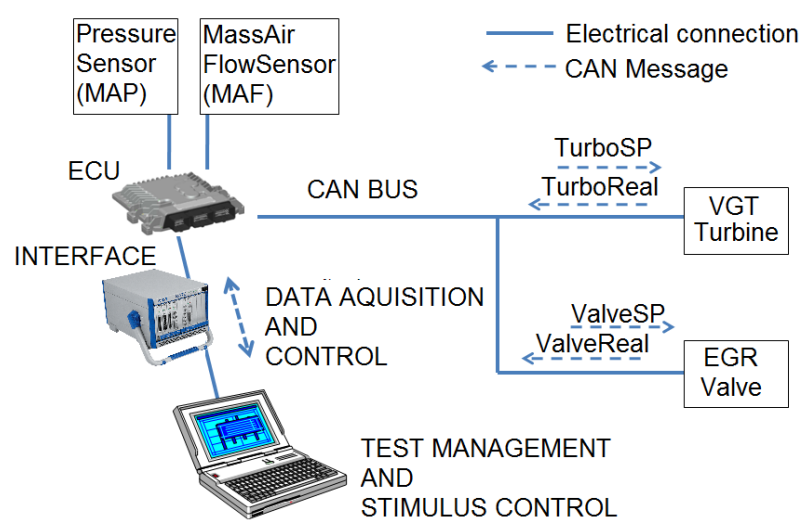

Figure 6: Architecture of ECU, airpath actuators and test management system.

- TurboSP is the CAN message transmitted by ECU to the VGT actuator requesting a specific position set point (from 0 to $100 \%$ );

- TurboReal is the CAN message transmitted by VGT actuator to inform the actual actuator position measured by its internal linear position sensor (from 0 to 100\%);

- ValveSP and ValveReal are also CAN messages with similar content (ECU transmits \% set point request position and valve returns the $\%$ actual position).

Computer runs specific control application software (ASCET from ETAS) that is connected to interface box (ES1000) and it's wired to ECU via dedicated ETK port. Test Management system can be seen below.

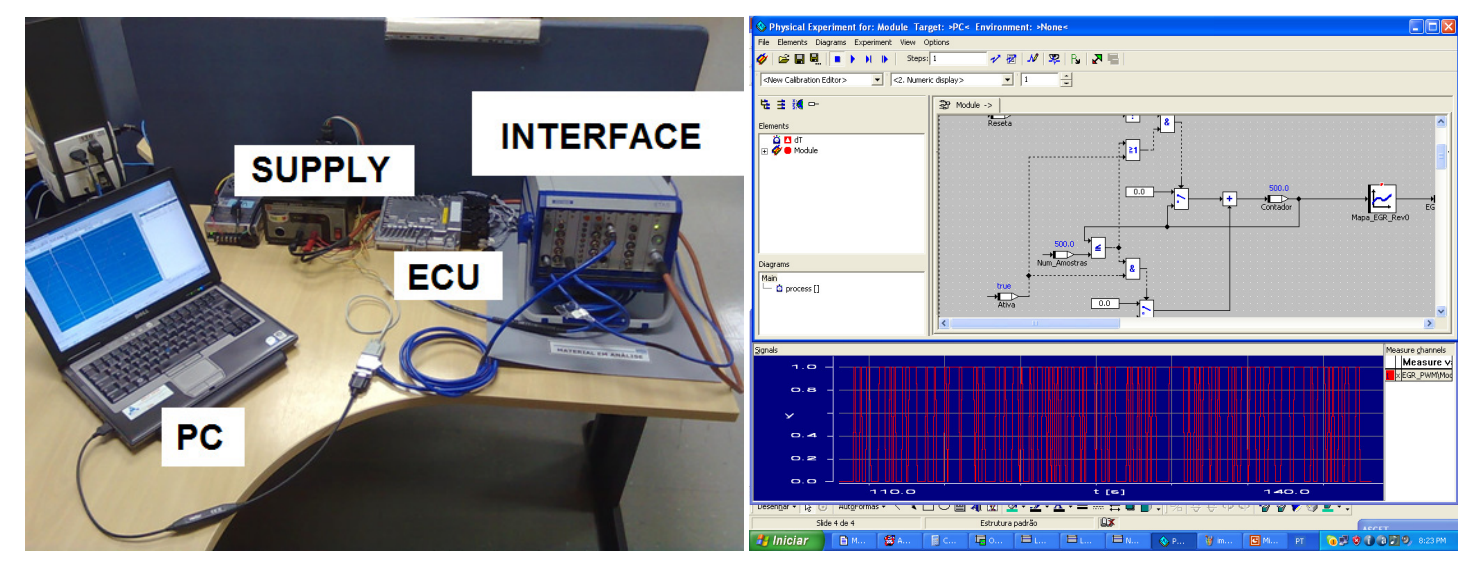

Figure 7: Test management system and software overview

\section{RESULTS}

\subsection{Engine stabilization}

After engine stabilization in the desired operating condition (2308RPM and 25\% of load) without any intervention the control parameters and sensor readings were recorded as indicated in Table 1.

Table 1: Average value taken from the stable engine operation.

\begin{tabular}{|l|l|l|}
\hline Parameter and sensors & Value & Unit \\
\hline Exhaust gas recirculation valve opening (EGR) & 15,00 & $\%$ \\
\hline
\end{tabular}




\begin{tabular}{|l|l|l|}
\hline Variable geometry turbine position (VGT) & 76,00 & $\%$ \\
\hline Intake manifold air pressure (MAP) & 1454 & hPa absolute \\
\hline Mass air flow (MAF) & 803 & Milligrams/stroke \\
\hline Engine speed & 2308 & RPM \\
\hline Engine load & 25 & $\%$ \\
\hline
\end{tabular}

\subsection{Linearity Evaluation}

To evaluate the linearity air pressure (MAP) with regards to the turbo position control (VGT) it was programmed in the test management system an individual VGT position sweep by small steps ( 1\% each step) with 10seconds delay for stabilization after each step increment. Test was repeated for the mass air flow (MAF) response with regards to the EGR valve opening. Both linearity check test results are seen on Figure 8.
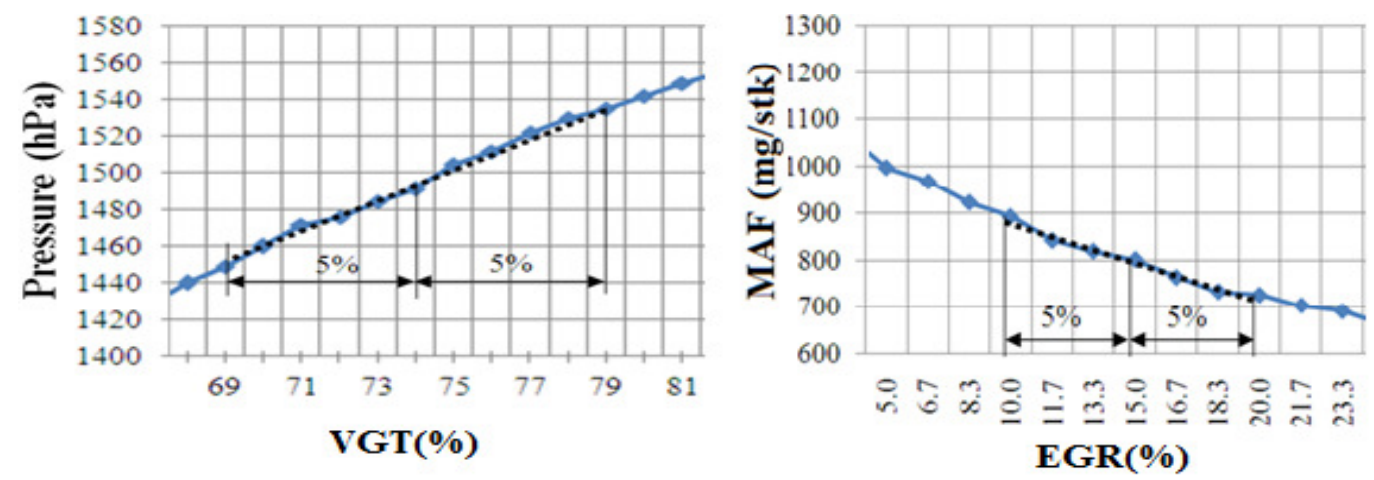

Figure 8: Mass air flow measurement as function of EGR valve opening.

It can be seen that there is an approximately linear behavior between EGR valve position control and measured mass air flow (MAF) of $+/-5 \%$ around stabilization point $(15 \%)$. An approximately linear behavior is also seen between pressure and VGT position of $+/-5 \%$ around stabilization point (76\%). These 2 tests confirm that the use of linear models is a valid approach for the airpath modeling around the selected operation point. To ensure stimulus only in the linear region the actuators are limited to the values indicated in Table 2.

Table 2: Min and max limits of each actuator

\begin{tabular}{|l|l|l|l|}
\hline Actuator & Min & Average & Max \\
\hline EGR & $10 \%$ & $15 \%$ & $20 \%$ \\
\hline VGT & $71 \%$ & $76 \%$ & $81 \%$ \\
\hline
\end{tabular}




\subsection{Step stimulus results}

With engine stabilized in the selected operation point the test management system was used to apply positive step stimulus ( $+5 \%)$ to each actuator at a time and the results were recorded with $10 \mathrm{~ms}$ recurrence and the result can be seen in Figure 9.

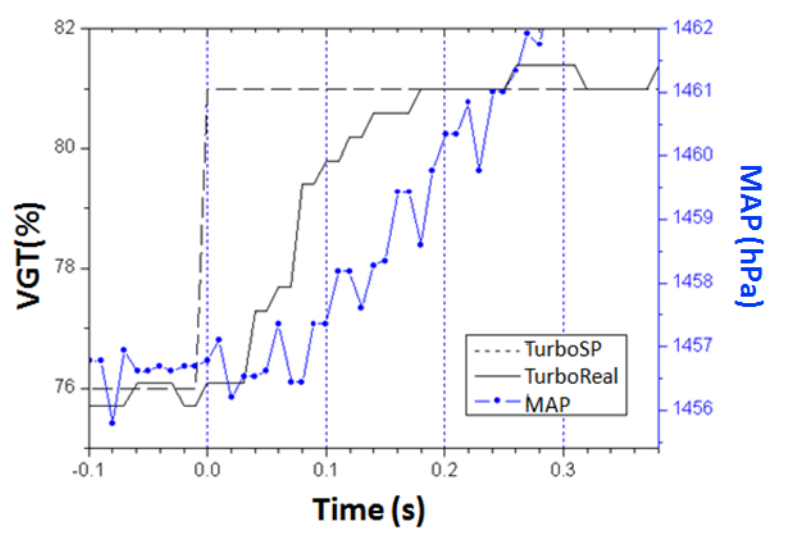

Figure 9: Step stimulus application (TurboSP) followed by the actuator (TurboReal) and the manifold pressure (MAP).

It's possible to see that the "TurboSP" requested an increasing step response and the turbine mechanic actuator reach the desired position with its characteristic displacement "TurboReal" causing the increase of the manifold pressure (MAP) that we can identify as positive effect (+) after the input positive stimulation. Between "TurboSP" and manifold pressure "MAP" can be noted an approximated 70ms time delay.

If we repeat the measurement for each input/output pair then we have delays and effect (signal contribution) seen in table below.

Table 3: Average measured transport delay and contribution for each model path

\begin{tabular}{|c|c|c|}
\hline Path & Transport delay & Effect (signal contribution) \\
\hline MAF(EGR) & $90 \mathrm{~ms}$ & Negative (-) \\
\hline MAF(VGT) & $50 \mathrm{~ms}$ & Negative (-) \\
\hline MAP(EGR) & $50 \mathrm{~ms}$ & Negative (-) \\
\hline MAP(VGT) & $70 \mathrm{~ms}$ & Positive (+) \\
\hline
\end{tabular}

Therefore we could detect the coupling effect between different systems as detected by YueYun [1] and also measured the transport delay between different input/output.

\subsection{Sinusoidal Frequency Response data analysis}

Test management system was prepared to apply a series of position requests to each actuator in sinusoidal shape at different frequencies. In each step the frequency increases ranging from 0.1 to $15 \mathrm{rad} / \mathrm{seconds}$ for VGT turbo and up to $40 \mathrm{rad} / \mathrm{seconds}$ for EGR valve. It was applied a sinusoidal signal with $+/-5 \%$ amplitude oscillation around the nominal operating point, (i.e. $76 \%+/-5 \%$ for the VGT Turbo and $15 \%+/-5 \%$ for EGR) during 10 seconds followed by another 10 seconds interval for plant stabilization. 
The sinusoidal signal will stimulate the actuator, propagate through the airpath and reach the plant output to be measured by ECU sensors. The plant gain would be determined by the amplitude ratio between plant output measured by the sensor and the actuator displacement amplitude. Calculating the plant gain when submitted by sinusoidal signals can be a repetitive task that requires considerable time. As an example it will be calculated the gain for only one frequency $(3.0 \mathrm{rad} / \mathrm{sec})$ for the Turbo stimulation and respective influence on pressure sensor reading. Peak-to-peak amplitude must be measured as seen on Figure 10 indicated by the dashed lines.

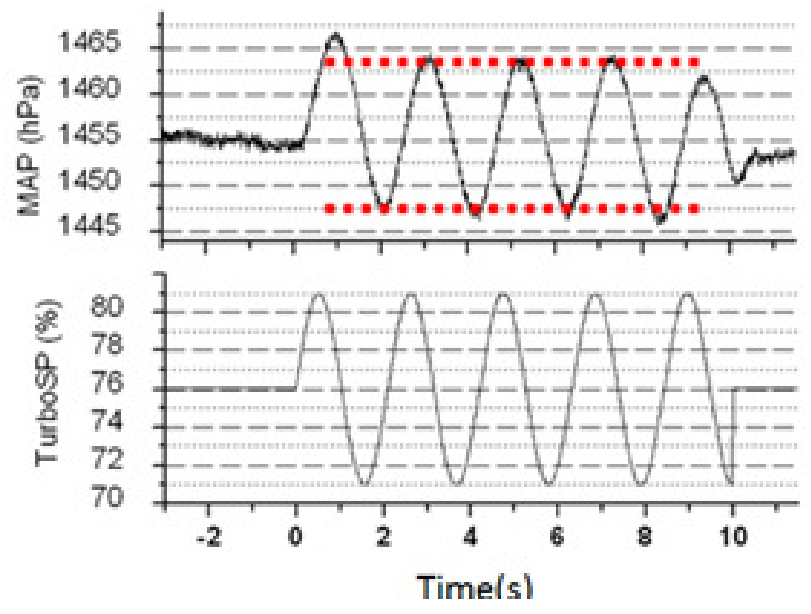

Figure 10: Sinusoidal stimuli (TurboSP) affected manifold pressure (MAP).

In the picture above we see that the manifold pressure (MAP) followed the position request oscillation (TurboSP) with its proper amplitude range. Graphically we could determine the gain by measuring the peak-to-peak amplitude of pressure oscillation ( dashed line) that is ranging from $1463 \mathrm{hPa}$ to $1447 \mathrm{hPa}$. Turbo actuator was stimulated by $10 \%$ peak-to-peak amplitude therefore plant gain can be calculated by:

$$
\text { Gain }=\frac{|1463-1447|}{|10|}=1,6\left[\frac{h P a}{\%}\right]
$$

This procedure shall be repeated for all other frequencies and the table in next section shows part of the results for example.

\subsection{Model Determination Using Frequency Response Method}

Plant measured gain values were calculated and they can be seen in Table 4 for example.

Table 4: Turbine oscillation and manifold pressure response

\begin{tabular}{|c|c|c|c|}
\hline $\begin{array}{c}\text { Frequency } \\
(\mathrm{rad} / \mathrm{s})\end{array}$ & $\begin{array}{c}\text { Turbine peak-to-peak } \\
(\mathrm{VGT})(\%)\end{array}$ & $\begin{array}{c}\text { Manifold pressure peak-to-peak } \\
(\mathrm{MAP})(\mathrm{hPa})\end{array}$ & $\begin{array}{c}\text { Plant gain } \\
(\mathrm{hPa} / \%)\end{array}$ \\
\hline 0,1 & 10 & 77 & 7,7 \\
\hline 0,6 & 10 & 54 & 5,4 \\
\hline 1,2 & 10 & 33 & 3,3 \\
\hline$\ldots$ & $\ldots$ & $\ldots$ & $\ldots$ \\
\hline
\end{tabular}


Plotting the plant gains in a di-log paper we obtain the graph seen in Figure 11 where the measured gains are the dots and the dashed red lines are the asymptotes that will be used to adapt the graph into linear s-domain transfer functions.

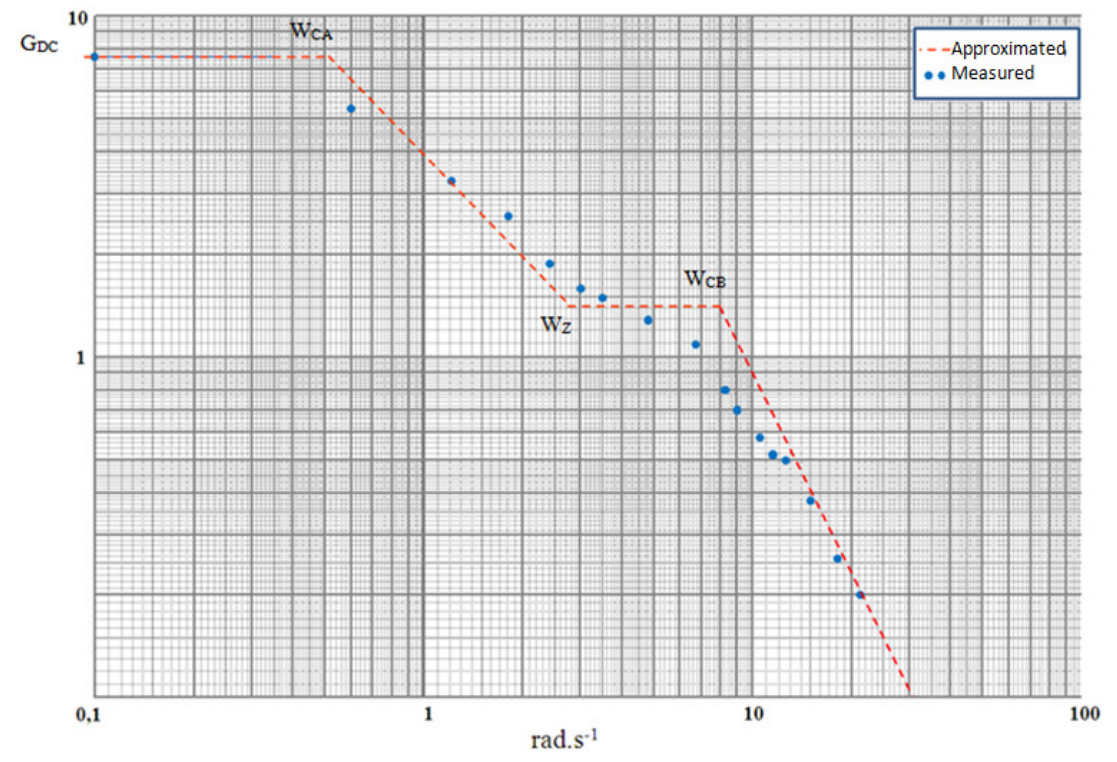

Figure 11: Plant gain per frequency (manifold pressure per turbine stimulation)

In the graph above (from left to right) there is a constant gain path $\mathrm{G}_{\mathrm{DC}}$ up to cutoff frequency $\mathrm{W}_{\mathrm{CA}}$ followed by an asymptote with $20 \mathrm{~dB} /$ decade decay up to $\mathrm{W}_{\mathrm{Z}}$ point. Another constant gain path up to $\mathrm{W}_{\mathrm{CB}}$ and then followed by an asymptote with $40 \mathrm{~dB} /$ decade decay. These notable points and asymptotes allow to build a mathematical description of the plant as seen in (8) without transport delay effect.

(8)

$$
G m a p_{-} v g t(j \omega)=\frac{G D C \cdot\left(\frac{1}{W z} j \omega+1\right)}{\left(\frac{1}{W c a} j \omega+1\right) \cdot\left(\frac{(j \omega)^{2}}{W c b^{2}}+\frac{2 . \zeta}{W c b} j \omega+1\right)}
$$

Notable points seen in Figure 11 (Frequencies: $\mathrm{W}_{\mathrm{Z}}, \mathrm{W}_{\mathrm{CA}}$ e $\mathrm{W}_{\mathrm{CB}}$; gain: $\mathrm{G}_{\mathrm{DC}}$ ) had their values determined graphically and the results can be seen in Table 5 .

Table 5: Notable frequency and gains

\begin{tabular}{|l|l|l|}
\hline Identification & Value & Unit \\
\hline $\mathrm{G}_{\mathrm{DC}}$ & $7,7 *$ & - \\
\hline $\mathrm{W}_{\mathrm{Z}}$ & 2,7 & $\mathrm{rad} . \mathrm{s}^{-1}$ \\
\hline $\mathrm{W}_{\mathrm{CA}}$ & 0,5 & $\mathrm{rad} . \mathrm{s}^{-1}$ \\
\hline $\mathrm{W}_{\mathrm{CB}}$ & 8 & $\mathrm{rad} . \mathrm{s}^{-1}$ \\
\hline
\end{tabular}

* Gain $\mathrm{G}_{\mathrm{DC}}$ is positive as detected in the step stimulus test

However the frequency response methodology doesn't allow obtaining the damping ratio $(\zeta)$ directly and it must be determined by hypothesis test. Some simulations comparing the pressure measured and the simulated pressure given by the model built including the transport delay were tried with different damping ratios $(\zeta)$ for comparison. Exactly the same input 
stimulus applied into the real turbine actuator test was also applied to the model under development and the results and measured pressure can be seen in Figure 12.

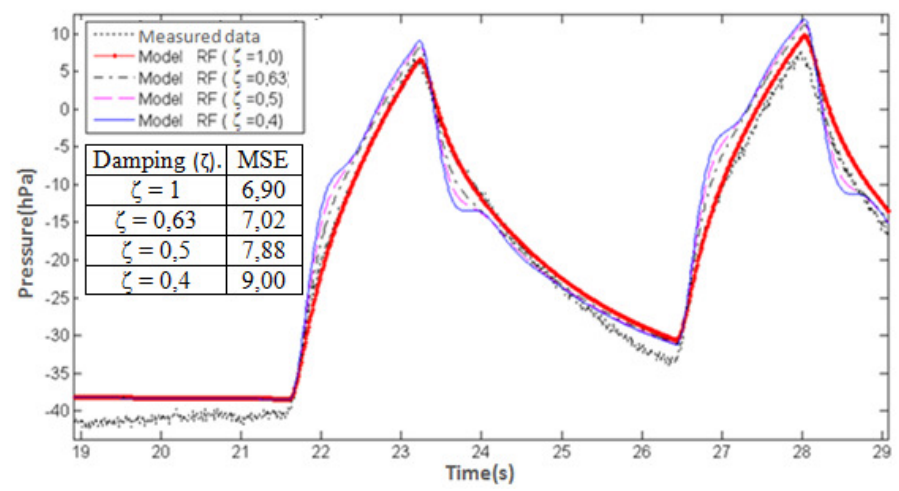

Figure 12: Comparison among manifold pressure (MAP) and four different models with different damping factors $(\zeta)$ and their respective MSE.

From the picture and MSE calculated values it's possible to see that unitary damping ratio $\zeta=$ 1 showed the best graphical matching to measured data and lowest calculated mean squared error (MSE). Therefore the model that best describes the manifold pressure (MAP) as function of VGT position is given by (9).

(9)

$$
G m a p_{-} v g t_{-} R F(j \omega)=\frac{7,7 \cdot\left(\frac{1}{2,7} \cdot j \omega+1\right)}{\left(\frac{1}{0,5} \cdot j \omega+1\right) \cdot\left(\frac{(j \omega)^{2}}{8^{2}}+\frac{2}{8} \cdot j \omega+1\right)}
$$

Repeating the method for all measured data then we obtain the following equations.

$$
\begin{gathered}
G m a p_{-} e g r_{-} R F(j \omega)=\frac{-16,2}{(j \omega+1) \cdot\left(\frac{(j \omega)^{2}}{10^{2}}+\frac{2}{10} \cdot j \omega+1\right)} \\
G m a f_{-} v g t_{-} R F(j \omega)=\frac{-1 \cdot\left(\frac{1}{0,1} \cdot j \omega+1\right)}{\left(\frac{1}{0,8} \cdot j \omega+1\right) \cdot\left(\frac{(j \omega)^{2}}{8^{2}}+\frac{2 \cdot 0,5}{8} \cdot j \omega+1\right)} \\
G m a f_{-} e g r_{-} R F(j \omega)=\frac{-20 \cdot\left(\frac{1}{7,4} j \omega+1\right)}{\left(\frac{1}{5,6} j \omega+1\right) \cdot\left(\frac{(j \omega)^{2}}{10^{2}}+\frac{2}{10} j \omega+1\right)}
\end{gathered}
$$

\subsection{Model Determination Using Autoregressive Exogenous Method (ARX)}

Data measured during engine test with PRBS stimulus will be used to identify an autoregressive (ARX) function to represent the engine airpath and it can be completely done using computer programs. Detailed steps to obtain the model of manifold pressure (MAP) as function of turbine actuator (VGT) are described as follow. 
We must have available the data digitally stored during the engine tests:

- VGT actuator position (\%)

- Manifold Pressure sensor (hPa)

- Time marker (seconds)

In this test it was adopted the $10 \mathrm{~ms} \pm 1,5 \mathrm{~ms}$ sampling rate and for clarification the first lines of the digital file can be seen on Table 6 .

Table 6: Sample of the PRBS data stored

\begin{tabular}{|l|l|l|}
\hline Time (s) & TurboSP (\%) & MAP \\
\hline 0,00132785 & 76 & 1453,9 \\
\hline 0,01030393 & 76 & 1453,9 \\
\hline 0,02091202 & 76 & 1454,0 \\
\hline$\ldots$ & $\ldots$ & $\ldots$ \\
\hline
\end{tabular}

First column is the time marker; middle column is the turbo actuator position followed by the measured pressure in the third column. Data plot is seen in Figure 13.

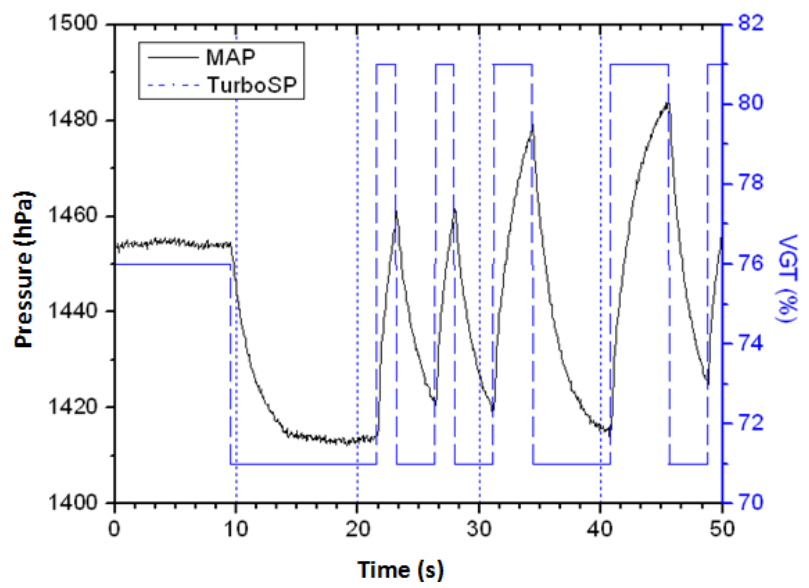

Figure 13: PRBS stimuli applied to the VGT actuator and manifold pressure (MAP).

It's possible to see the turbo actuator moving according to PRBS signal stimulation with +/$5 \%$ amplitude around nominal operation point $(76 \%)$ followed by manifold pressure dynamic response. With the digital computer files available the analysis can be done in Matlab application. In such program the command sequence required to obtain ARX model can be seen in Table 7.

Table 7: MATLAB commands to obtain ARX model

\begin{tabular}{|l|l|}
\hline Command & Function \\
\hline $\begin{array}{l}\text { data=DDATA(plant_output,stim } \\
\text { ulus,Tsampling) }\end{array}$ & $\begin{array}{l}\text { Creates DATA-MATLAB object with plant } \\
\text { output (MAP), input (VGT) and 10ms sampling } \\
\text { time }(0.01 \mathrm{sec}) .\end{array}$ \\
\hline $\begin{array}{l}\text { data_no_trend = DETREND } \\
\text { (data) }\end{array}$ & Remove linear trends. \\
\hline $\begin{array}{l}\text { model_arx=ARX } \\
\text { (data_no_trend, [P Z T], 'focus', } \\
\text { 'simulation') }\end{array}$ & $\begin{array}{l}\text { Determine discrete ARX transfer function with Z } \\
\text { (zeros), P(poles), T(transport delay). It specifies } \\
\text { also that program should focus to obtain model } \\
\text { for simulation purposes. }\end{array}$ \\
\hline
\end{tabular}


model_continuous $=$

D2C(model_arx)
Converts discrete function into continuous sdomain.

As the previous frequency response analysis showed existence of 3 poles and 1 zero then it's a good indication of the ARX model order therefore it was used 3 poles $(\mathrm{P}=3), 2$ zeros $(\mathrm{Z}=2)$ and transport delay measured of $70 \mathrm{~ms}(T=7$, seven sampling periods of $10 \mathrm{~ms}$ each). Different number of poles and zeros can be tried to find the best compromise between model complexity and accuracy. The resulting model for manifold pressure is seen in (13).

$$
G m a p_{-} v g t_{-} A R X(S)=\frac{7,566 S^{2}+348 S+1559}{S^{3}+56.21 S^{2}+400,7 S+206.4}
$$

The same procedure described above was repeated for the other models resulting in the plant modeling seen in Table 8.

Table 8: Transfer functions obtained by ARX modeling

\begin{tabular}{|l|l|l|l|}
\hline Model & Poles & Zeros & Transfer function \\
\hline MAP(VGT) & 3 & 2 & $\frac{7,566 S^{2}+348 S+1559}{S^{3}+56.21 S^{2}+400,7 S+206.4}$ \\
\hline MAP(EGR) & 3 & 2 & $\frac{-0,168 S^{2}-33.76 S-1806}{S^{3}+94,21 S^{2}+198,6 S+113,4}$ \\
\hline MAF(VGT) & 3 & 2 & $\frac{-23,6 S^{2}-8235 S-7,5 e 5}{S^{3}+261,3 S^{2}+1392 e 4 S+7,785 e 4}$ \\
\hline MAF(EGR) & 3 & 2 & $\frac{-36,23 S^{2}-1,13 e 4 S-9,295 e 5}{S^{3}+224,1 S^{2}+9927 S+4,474 e 4}$ \\
\hline
\end{tabular}

3.6 Model Determination Using Statistical Learning

Statistical and modeling know-how are not required knowledge with the NARX-GP tool (ASCMO). No parameterization is needed to model the system as it is focused on finding automatically the most likely behavior of the plant using a set of functions. Once the data is ready and available the next step is load it in the Software and define the inputs and outputs:

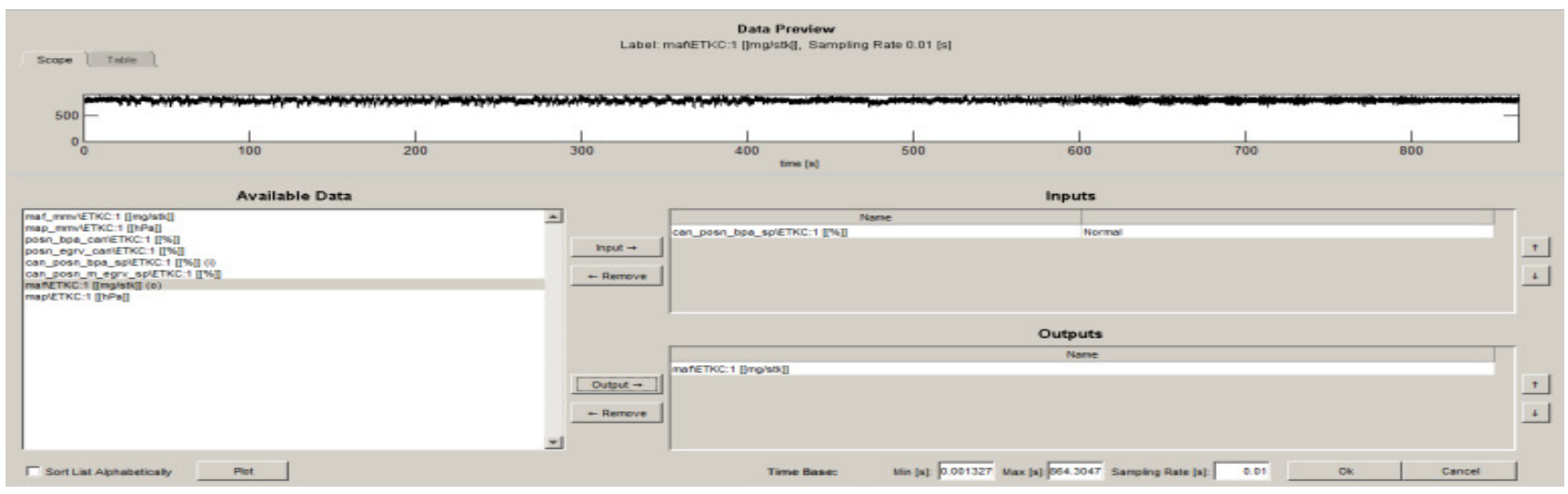

Figure 14: Setting Inputs and Outputs of the Model 
Picture above shows an overview of the tool main screen where the input and output variables used for modeling are selected and measured data can be evaluated. Tool offers other features like down sampling and exclusion of outliers points.

To obtain the model the following settings are concerned:

- The Modeling algorithm;

- The Feedback (NARX) structure;

- Dimensionality Reduction;

For instance, the MAP (VGT) Model, the following settings have been selected.

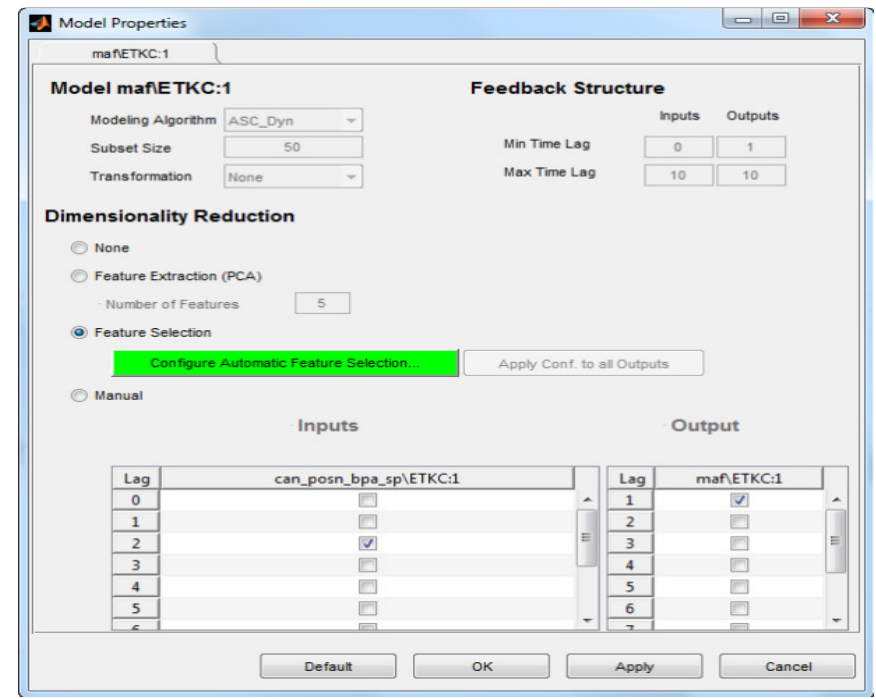

Figure 15:Model Properties setup

With these adjustments (it was set automatic selection) the Model is trained and the results can be analyzed by the tool (e.g.; Phase Plot). The last step is to export the model to the desired format (SIMULINK, MATLAB script or C CODE). The SIMULINK model is shown in figure below.

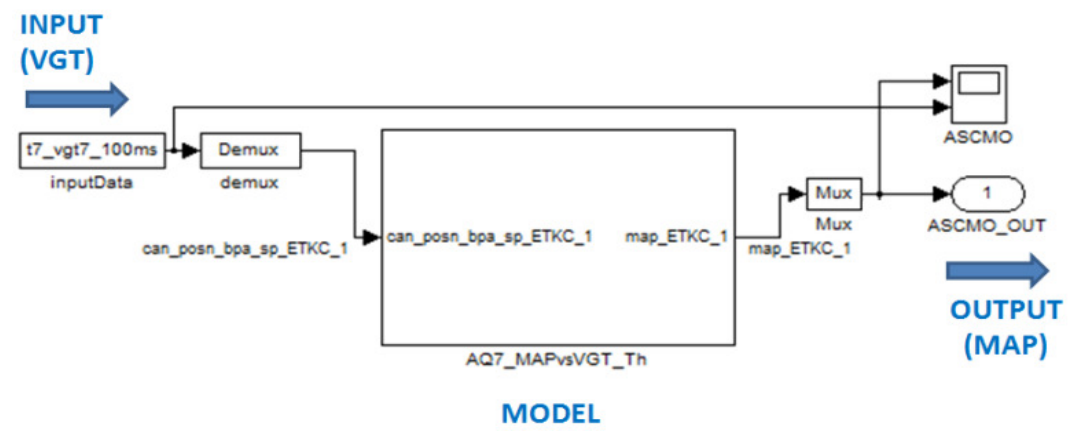

Figure 16 - MAP(VGT) NARX-GP output model in SIMULINK.

In the figure above it's possible to see the model input (VGT position) on the left side of diagram and the output (MAP) at the right side. This is the main diagram while the low level model is enclosed in the box named "MODEL" and its detailed calculation is seen below (time discrete format). 


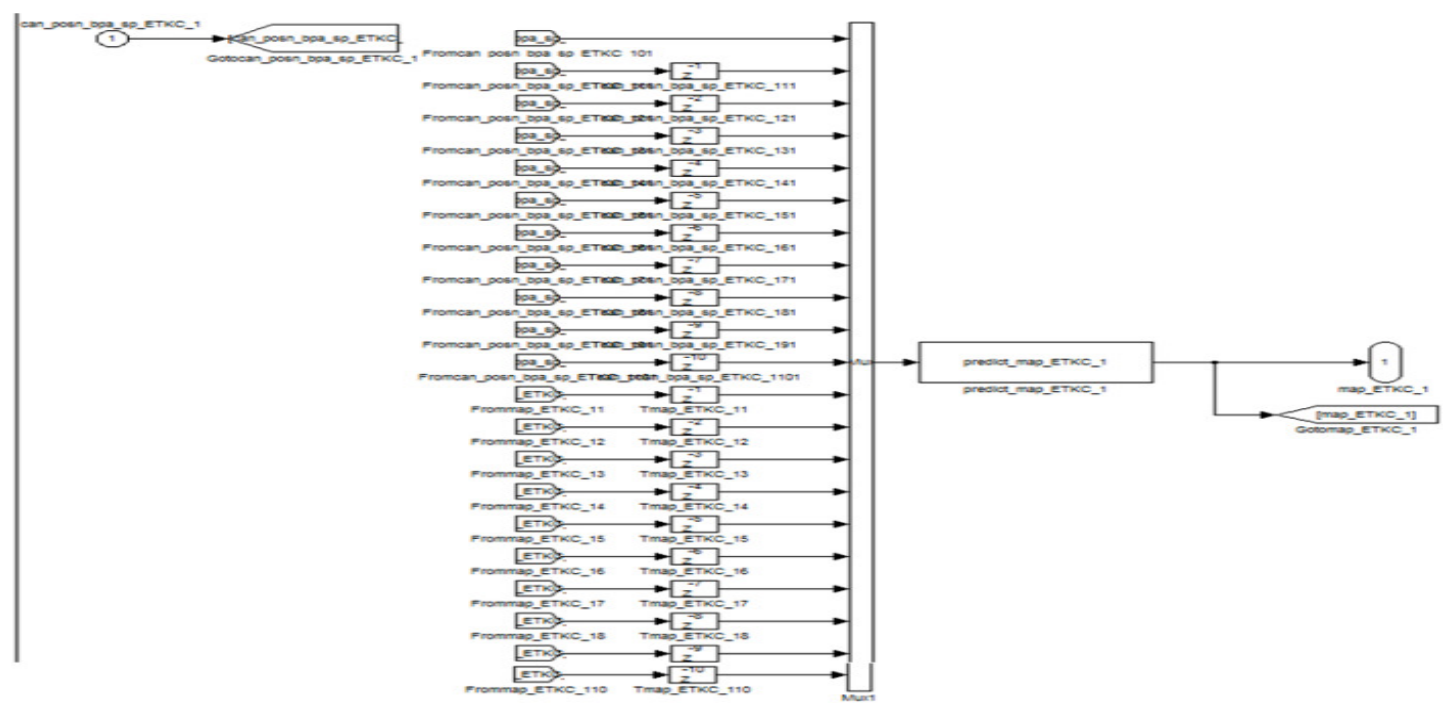

Figure 17: NARX-GP detailed model calculation in SIMULINK

The complexity of the SIMULINK block seen above in the picture depends on the number of inputs/outputs and number of measured points considered into the model.

\subsection{Models Representation in SIMULINK}

To be able to simulate the airpath model in SIMULINK then four input-output models must be integrated considering their contribution to the final sensed value (MAP or MAF) depending on the actuator position (VGT or EGR) and the respective transport delay measured. The result is the sum of contributions for each variable. The engine airpath plant representation in SIMULINK block diagram should look like seen in Figure 18.

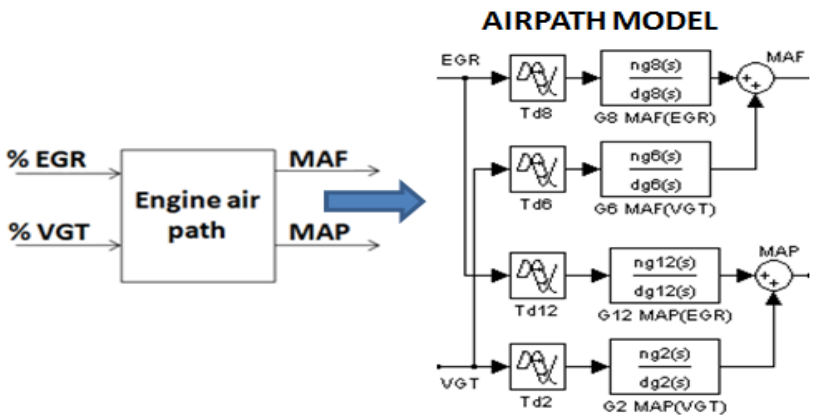

Figure 18: Engine airpath plant model

The block diagram above is made of four transport delay and four continuous transfer function blocks. The plant output is the sum of effects from both actuators EGR and VGT over the pressure (MAP) and flow (MAF).

As the model is valid at one engine operation point then each one of the four models must be adjusted for the operation points seen on Table 1. Final diagram should look like seen below. 


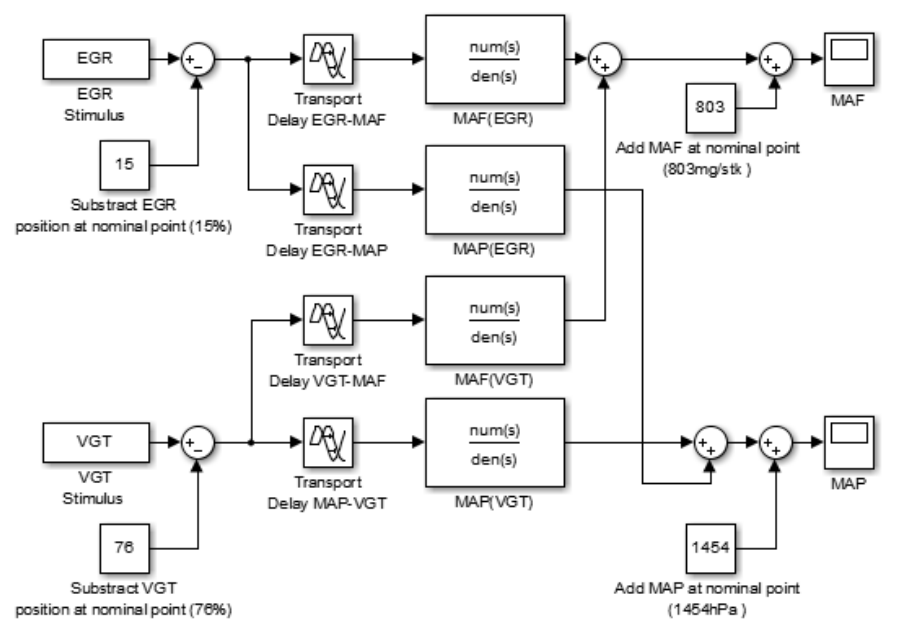

Figure 19: ARX and FR model representation

To adjust model around the chosen operation point then it's needed to subtract the average value of the actuator in the nominal operating point (EGR $=15 \%$ and $\mathrm{VGT}=76 \%$ ) as seen in the picture above. The resulting actuator relative position is applied to the transport delay blocks that are followed by the respective dynamic transfer function. Finally the dynamic models contribution are added accordingly and resulting output needs to be added again by the average pressure and air mass measured to make it relative to the nominal point where the model is valid $(\mathrm{MAF}=803 \mathrm{mg} / \mathrm{stk}$ and $\mathrm{MAP}=1454 \mathrm{hPa})$.

NARX-GP tool output model doesn't require additional handling to build the global model as it's already in a block format ready to implement into a simulation environment that considers the nominal operation point characteristics.

\section{METHODOLOGIES COMPARISON}

It could be seen in the developments above that each modeling method uses different resources and require different tests. A summary of methods is listed below for a quick review.

\subsection{Frequency Response}

Required step stimulus to determine the transport delay ; Several frequency stimulus for plant gain calculation; Plot gains in di-log paper for asymptote definition; Linear equation determination and in some cases the damping ratio also was defined by test.

\subsection{Auto Regressive Exogenous (ARX)}

Required step stimuli to determine the transport delay ; PRBS signal stimulation to produce base data for modeling followed by computer calculation in MATLAB to obtain the discrete ARX equations and additional MATLAB commands can convert it into continuous s-domain model when needed.

\subsection{Statistical Learning (NARX-GP tool)}

Required the PRBS recorded data base and some user-selections to determine model accuracy and dimension/size. Model accuracy is determined online by the tool using methods like 
"Leave one out" and it also offers option to check model accuracy using data that was not previously used to generate the model. Tool can guide user to build specific DoE (design of experiments) but DOE is not required to build the model. Model output is delivered in discrete time format.

Table 9 - Overview of stimulus used for each methodology.

\begin{tabular}{|l|c|c|c|c|c|}
\hline Methodology & Step & Sinusoidal & PRBS & Damping ratio $(\zeta)$ & Gain Plot \\
\hline RF & Y & Y & & Y & Y \\
\hline ARX & Y & & Y & & \\
\hline NARX-GP & & & Y & & \\
\hline
\end{tabular}

Note: "Y" Stimulus used

In the table it's listed the stimulus used for each modeling technique in this work. Here we note that frequency response method used the higher amount of signals and tasks to determine the model, followed by ARX that used only PRBS and step stimulus. Finally the NARX-GP tool could achieve the model without need for step stimulation.

\subsection{Model Results Comparison}

Additionally to the methodologies comparison the model performance was checked. All models were migrated to same SIMULINK environment for comparison under same basis and result is seen in the next figures.

\subsection{Simulation Results}

After complete simulation of the three engine airpath models obtained by different methodologies (Frequency Response, ARX, NARX-GP/Statistical learning) they were compared graphically and also by the Mean Squared Error (MSE) of each model as follow.
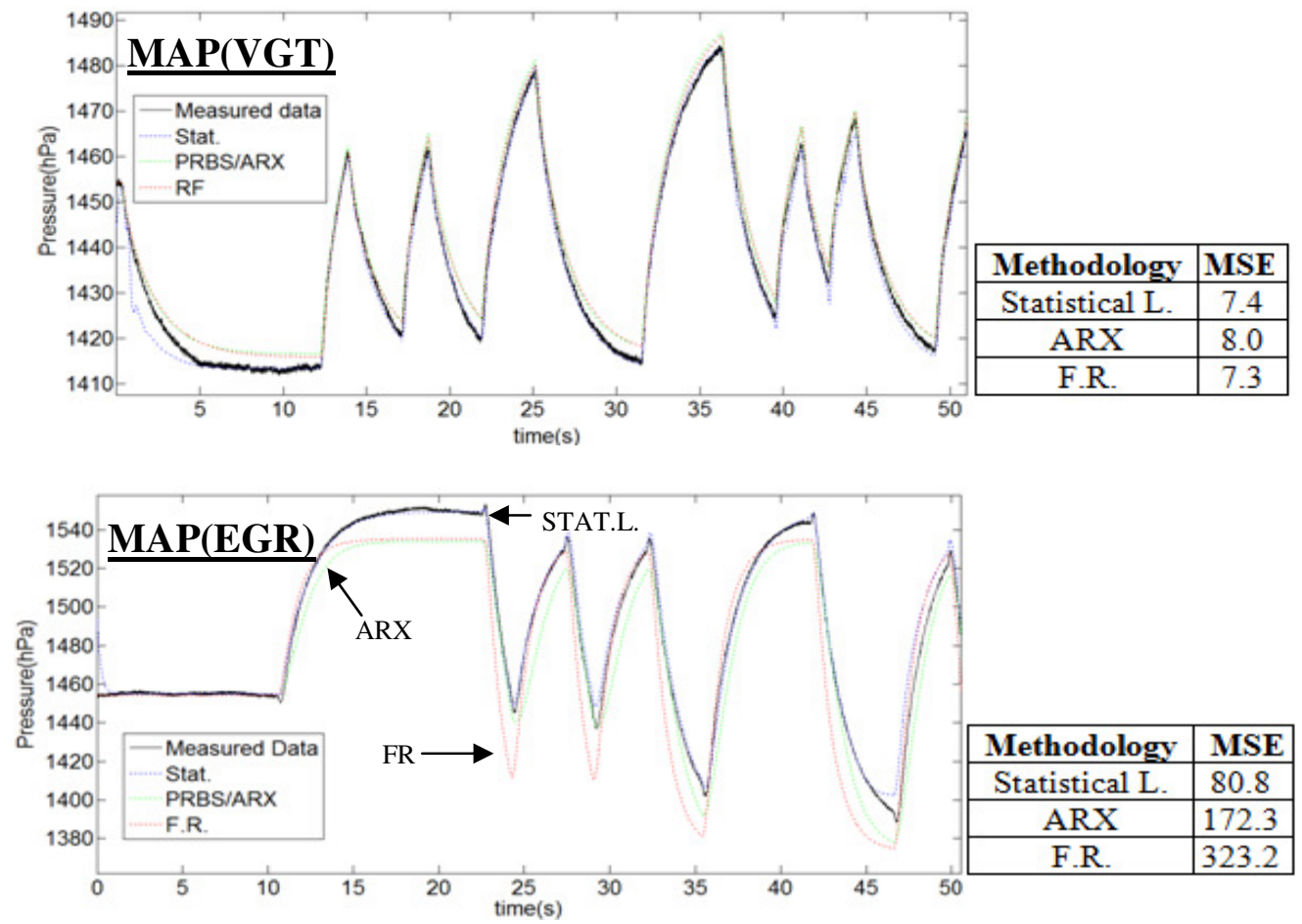

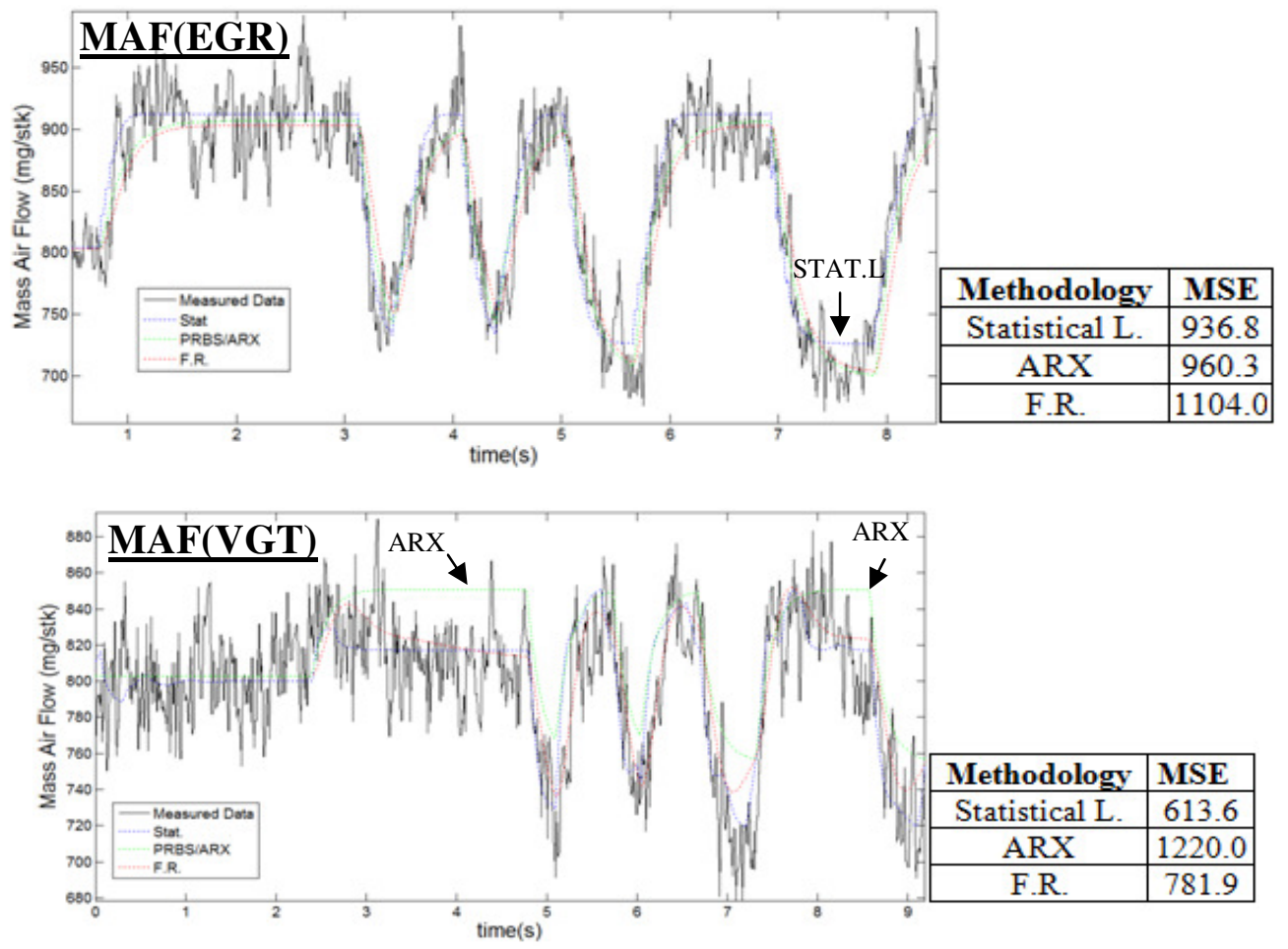

Figure 20: Plot of measured data, NARX-GP/Statistical learning, ARX and Frequency Response (F.R.) models as function of each input and respective Mean Squared Error (MSE).

From the pictures we see that for the MAP(VGT) simulation three methods achieved good performance as they can track the signal very close and they have similar MSE values.

For the MAP(EGR) simulation the ARX and RF models have a permanent deviation from the measured data while the NARX-GP/Statistical learning could track the signal closer. NARXGP/Statistical learning tool achieved much lower MSE.

For the MAF(EGR) simulation three methods achieved good performance as they can track the signal very close and they have similar MSE values in average.

For the MAF(VGT) simulation the Statistical learning and RF models have good average signal tracking and they have similar MSE values. The ARX model presented a persistent signal deviation that was also detected by the high MSE value.

\section{CONCLUSION}

Three mathematical modeling methodologies were described in details while they were used to build airpath models of a diesel engine to use in Hardware in the Loop simulator.

Modeling methodology and performance were compared and can be summarized as follow:

Frequency Response methodology was found complex in this work as it used step and sinusoidal test data stimulus, damping ratio tests and gain plots to produce the models. Manual work was applied in all stages but as result the models have average good quality to represent the plant dynamics. 
Auto Regressive Exogenous (ARX) method is much simpler when comparing to Frequency Response because it used only step and PRBS test data stimulus. Manual work was applied only to visually determine the transport delay. Models were obtained after computational work in Matlab and models have good data adherence in average but in one case (MAF as function of VGT position) airpath dynamic couldn't be well represented. In this case the zero allocation was better done by FR and NARX-GP methods.

Statistical Learning method which is the key algorithm of a commercial tool could produce good models using only PRBS stimulus test data available. An advantage of this tool is that transport delay visual determination was not required as an input for the modeling. As result this methodology produced accurate and good models in very short time and demanding almost no modeling know-how or adjustment to nominal operation point. Comparing to other two approaches and the evaluation based on MSE index the tool showed good model accuracy.

This work considered only modeling in a fixed point (2308RPM at 25\% of load) assumed as linear over a certain range. Multiple operating points modeling is possible but it requires additional investigations and other techniques in addition (e.g. engine operation range scheduling) to accomplish the full engine airpath modeling.

\section{REFERENCES}

[1] YUE-YUN, W.; HASKARA, I.; YANIV, O. Model-Based quantitative feedback control of EGR rate and boost pressure for turbocharged diesel engines. In: 2008 American Control Conference. Seattle, Washington, USA, 2008. p.291-297.

[2] CHAUVIN, J. et al. Airpath strategy for experimental transient control of a diesel HCCI engine. Oil ; Gas science and technology, v.62, n.4, p.483-491, 2007.

[3] ALFIERI, E. et al. Model-based feedback control of the air-to-fuel ratio in diesel engines based on an empirical model. In: Proceedings of the 2006 IEEE international conference on control applications. Munich, Germany, 2006. P.509-514.

[4] OGATA, Katsuhiko. Instrumentation and control series: Modern Control Engineering. New Jersey. Prentice Hall. 1970.

[5] UENO, M.; IWADARE, M.; ADACHI, S. Multi-variable airpath management for a clean diesel engine using model predictive control. In: SAE World Congress \& Exhibition, April 2009, Detroit, MI, USA, SAE PAPER 2009-01-0733, 10p.

[6] ORTNER, Peter; DEL RE, Luigi. Predictive control of a diesel engine airpath. IEEE Transactions on control systems technology, v.15, n.3, p. 449-456, 2007.

[7] LOUNGHBOUROUGH University \& CATERPILLAR In. Using a Statistical Machine Learning Tool for Diesel Engine Air Path Calibration

[8] WERKEMA, M. C. ; AGUIAR, S. Análise de Regressão. Belo Horizonte: Werkema editora, 1996.

[9] ETAS GmbH. ETAS ASCMO Dynamic v4.5 User's Guide, 2014. 